\title{
Evaluación de Diferentes Metodologías de Aprendizaje Activo desde el Punto de Vista del Estudiante en la Asignatura Genómica del Grado de Biotecnología
}

\section{Santiago Vilanova y José Gadea}

Departamento de Biotecnología, Universitat Politècnica de Valéncia, Camino de Vera s/n 46022, Valéncia. jgadeav@ibmcp.upv.es sanvina@upvnet.upv.es

\begin{abstract}
Biotechnology is a discipline where autonomous learning can be easily implemented. The speed at which strategies and technologies are developed makes mandatory a learning method more focused in "how to learn" than in "what to learn". The objective of this study is to evaluate the student's level of satisfaction regarding the learning strategies applied in the subject "Genomics" of the UPV Biotechnology Degree. Among these methodologies, we have used project-based learning, case studies and flip-teaching. Results seem to indicate a high percentaje of satisfaction when these methodologies are applied.
\end{abstract}

Keywords: autonomous learning, flip-teaching, active learning, project, biotechnology

\footnotetext{
Resumen

La Biotecnología es una disciplina donde puede implementarse el aprendizaje autónomo. La velocidad a la que las tecnologías y estrategias están cambiando hace necesario un aprendizaje más enfocado a "cómo aprender", más a "qué aprender”. El objetivo de esta comunicación es evaluar el grado de satisfacción de los estudiantes en referencia a los métodos docentes aplicados en la asignatura Genómica del Grado de Biotecnología de la UPV. Entre estas metodologías, hemos aplicado aprendizaje basado en proyectos, estudio de casos y clase inversa. Los resultados parecen indicar que un alto porcentaje de alumnos está satisfecho con un aprendizaje basado en estas metodologías.
}

Palabras clave: aprendizaje autónomo, clase inversa, aprendizaje activo, proyectos, biotecnología 


\section{Introducción}

El aprendizaje autónomo implica la intervención del estudiante en el establecimiento de sus objetivos, procedimientos, recursos, evaluación y momentos de aprendizaje (Crispin et al, 2011). Estas estrategias confían en un aprendizaje activo, eliminando el sistema pasivo basado en clases magistrales, pone énfasis en un aprendizaje más profundo y un mejor entendimiento del tema tratado, aumenta la responsabilidad por parte del estudiante e incrementa su sentido de autonomía, y al mismo tiempo, proporciona un ambiente donde la relación profesor-alumno puede ser replanteada.

Entre las estrategias que se plantean para ejercitar el aprendizaje autónomo, el diseño de proyectos en equipos de estudiantes es la que hemos seleccionado para esta experiencia docente. El aprendizaje basado en proyectos es una metodología que permite a los alumnos adquirir los conocimientos y competencias clave en el siglo XXI mediante la elaboración de proyectos que dan respuesta a problemas de la vida real. El trabajo en equipos, por otra parte, refuerza otras competencias transversales, ya que actualmente las organizaciones requieren de personas que sepan administrarse dentro de los equipos de trabajo (Northwest Regional Educational Laboratory, 2006).

La Biotecnología es una disciplina donde puede y debe implementarse el aprendizaje autónomo. La inmensidad de avances que se están produciendo en esta ciencia, y en concreto en Genómica, la asignatura donde hemos implementado esta iniciativa, hace imposible cubrir todos los conocimientos que un estudiante podría requerir para la práctica profesional. Por otra parte, la velocidad a la que las tecnologías y estrategias están cambiando, quedándose obsoletas en varios años, hace necesario un aprendizaje más enfocado a "cómo aprender", más a "qué aprender". Este aprendizaje debe incluir la búsqueda de todas las posibilidades existentes para solucionar un determinado problema y la toma de decisiones para seleccionar una determinada estrategia de trabajo, sin tener la certeza de que sea la única manera de abordar dicho problema. También implica enfrentarse a situaciones en las que "no se sabe todo" y "no se controla todo", un escenario al que los estudiantes no están acostumbrados. Finalmente, un proyecto biotecnológico real será multidisciplinar, por lo que un aprendizaje cooperativo donde se elimine la excesiva competitividad existente entre los alumnos de Biotecnología podría ser beneficioso para ellos a largo plazo.

\section{Objetivos}

Desde el curso académico 2012-2013, llevamos proponiendo diseños de proyectos en Genómica como ejercicio de aprendizaje autónomo a los estudiantes de Biotecnología de tercer curso de la Universidad Politécnica de Valencia. Los proyectos consisten en emular los diferentes pasos que un profesional en biotecnología deberá realizar al enfrentarse a un proyecto de estas características, búsqueda bibliográfica, selección de tecnologías, toma de decisiones, diseño experimental, localización de reactivos y equipamiento, presupuesto económico y cronograma de realización, basándose en cada punto en estudios similares que ya estén publicados en contextos diferentes. Con esto, los alumnos se enfrentan a la realidad de un proyecto de investigación (o un proyecto empresarial), que requiere no sólo 
un conocimiento teórico de un ámbito concreto de la Biotecnología, sino tener en cuenta muchos otros factores que condicionan el éxito real del proyecto.

Por otra parte, este curso 2016-2017 hemos iniciado una estrategia docente de clase inversa, con tareas planteadas a los estudiantes, cuyo objetivo es intentar que se consolide y/o se sepa aplicar la información teórica que los estudiantes reciben con clases magistrales distribuidas selectivamente a lo largo de la asignatura. Ésta consta de 5 bloques temáticos, cada uno de las cuáles introduce, desarrolla y ejemplifica la aplicación de un subcampo de la Genómica. Para ello los estudiantes realizan tareas en Google Drive, que alternan entre lectura de artículos científicos seleccionados, visionado de videos o búsqueda de información en la web.

La presente comunicación pretende resumir esta experiencia docente, detallando las conclusiones a las que tanto profesores como estudiantes hemos llegado.

\section{Desarrollo de la innovación}

\subsection{Encuestas de evaluación}

Mediante el uso de encuestas enfocadas a evaluar la experiencia de los estudiantes en esta iniciativa, se contactará a los estudiantes de Biotecnología del curso 2016-2017, y se elaborarán resultados que permitan la interpretación detallada. Las encuestas pretenden desvelar su grado de satisfacción con este tipo de docencia, la repercusión de este tipo de aprendizaje en el conocimiento de la asignatura, la originalidad y valor añadido de los mismos, relación profesor-alumno, etc. Las encuestas Se realizaron online utilizando la herramienta de formularios de Goole Drive (https://www.google.com/intl/es_ALL/drive/).

La encuesta consta de 21 ítems con la siguiente escala de evaluación de Likert (Briones, 1995) : Muy en desacuerdo(1), En desacuerdo(2), Indiferente(3), De acuerdo(4) o Muy de acuerdo(5). Los ítems se subdividen en cuatro secciones: a) estructura general de la asignatura, b) la experiencia en la realización de casos prácticos, c) la experiencia en la realización de tarea de clase inversa, y d) la experiencia en la realización de trabajos/proyectos de investigación

El cuestionario fue el siguiente:

\section{(EA) Estructura general de la asignatura}

EA1. Me ha resultado más EFECTIVA que la clase tradicional (profesor explicando y alumnos atendiendo) para entender los contenidos y su aplicación.

EA2. Me ha resultado más ENTRETENIDA que la clase tradicional (profesor explicando y alumnos atendiendo) para entender los contenidos y su aplicación.

EA3. Me ha ayudado a COMPRENDER contenidos en lugar de MEMORIZARLOS

EA4. Me ha ayudado a SABER APLICAR contenidos en lugar de MEMORIZARLOS

EA5. Hace que el profesor esté más implicado en la docencia, y por tanto interacciono más con él.

EA6. En un plan de estudios ideal sin tanta sobrecarga, recomendaría este tipo de docencia a otras asignaturas. 
EA7. Respecto a las otras asignaturas con las que se ha compartido en el año ¿Has dedicado más o menos tiempo de estudio a GENOMICA?

\section{(CP) Casos Prácticos}

CP1. Me han ayudado a CONSOLIDAR los contenidos teóricos

CP2. Me han ayudado a ver cómo se APLICA la teoría en la realidad

CP3. Me han quitado el miedo a enfrentarme a artículos científicos

CP4. Me han confundido más que ayudado

\section{(T) Tareas}

T1. Me han servido de BASE para la materia que se iba a explicar en clase, si son anteriores, o de CONSOLIDACIÓN, si son posteriores

T2. Me han quitado mucho tiempo de estudio

T3. Siendo sincero/a...¿Qué porcentaje de las tareas has contestado como si fuera a ser puntuada como una pregunta de examen?

\section{(P) Proyectos}

P1. Me han ayudado de discriminar entre información más relevante y menos relevante para mis objetivos

P2. Me han ayudado a darme cuenta de que puedo seguir adelante sin entender todos los detalles de un proyecto

P3. Me han ayudado a unir información proveniente de diferentes fuentes para resolver problemas específicos

P4. Me han ayudado a mejorar mis habilidades en estructurar la información escrita y oral

P5. Me han ayudado a mejorar mis habilidades en trabajar con otros en grupo

P6. Me han ayudado a mejorar mis habilidades en organizar y planificar mi tiempo

P7. Me ha motivado especialmente ver que soy capaz de crear un proyecto que nadie ha planteado hasta entonces.

\subsection{Análisis de datos}

Los datos obtenidos a partir de las encuestas se analizaron mediante la aplicación hojas de calculo de Google Drive.

Por otra parte, el análisis Heatmap se realizó mediante el programa online Clusvis (Metsalu et al. 2015; http://biit.cs.ut.ee/clustvis/). Los clusters dentro filas y columnas se calcularon usando las distancias euclideas y el método de ligamiento completo respectivamente.

\section{Resultados}

Disponemos actualmente de más de 70 encuestas realizadas con el objetivo de evaluar si la experiencia había sido positiva, y con objeto de mejorarla, donde las sugerencias de los estudiantes sean tenidas en cuenta. Esto representa el $70 \%$ de los estudiantes matriculados este año, por lo que consideramos la encuesta significativa. Un $24 \%$ de los encuestados 
están matriculados en el grupo de Alto Rendimiento Académico del Grado en Biotecnología, y un $76 \%$ en el grupo que se imparte en castellano.

A continuación pasamos a detallar los resultados más relevantes obtenidos en este proyecto:

\subsection{Estructura de la asignatura.}

Los estudiantes fueron preguntados si la estructura de la asignatura, de modo general (incluyendo tareas de clase inversa, clases magistrales alternas, y realización de trabajos de investigación, así como su división temática en casos, les ayudaba a comprender y/o a saber aplicar los contenidos en lugar de a memorizarlos, así como si este tipo de docencia les parecía más efectiva respecto a la docencia clásica de clase magistral, y si les parecía más entretenida. Finalmente, se les preguntaron aspectos generales como si recomendarían este tipo de docencia en un plan de estudios ideal sin tanta carga académica, y si habían dedicado más o menos tiempo de estudio a esta asignatura como consecuencia del tipo de docencia.

A rasgos generales, un $35 \%$ de los estudiantes consideró esta estructura más efectiva que la clase tradicional (EA1 Fig 1.), y hasta un 68 \% la consideró más entretenida (EA2 Fig 1.), lo que indica que la tendencia general es positiva. No obstante, llama la atención el porcentaje de estudiantes que aún se decantan por un aprendizaje más clásico, ya que el 20 $\%$ consideran esta estructura más inefectiva, y un $40 \%$ no ven ninguna ventaja respecto al aprendizaje tradicional (EA1 Fig 1.). En un futuro, pretendemos estudiar en detalle este grupo de alumnos, para intentar evaluar cuántos de ellos son indiferentes ante todo en cualquier caso, y cuántos de ellos prefieren el sistema clásico. Ante esto, hay que recalcar que muchos de ellos pueden preferir un sistema de memorización pasiva, al que ya están habituados desde pequeños, y que les genera unos mejores rendimientos en cuanto a notas, frente a un nuevo sistema que les obliga a implicarse activamente en el aprendizaje. Pensamos que estos últimos necesitan que les expliquemos las ventajas del aprendizaje autónomo para conseguir su implicación.

Por otra parte, la tendencia también es muy positiva en cuanto a si el método docente les ha ayudado a comprender y saber aplicar mejor los conceptos de la asignatura (EA3 Fig 1. y EA4 Fig 1.). Un $52 \%$ considera positiva la experiencia, frente a un $30 \%$ que no ve diferencia entre este método y el aprendizaje clásico. Sólo un $13 \%$ considera la experiencia negativa. Igual que antes, es el grupo intermedio el que conviene estudiar con más detalles para saber cuántos de estos estudiantes prefieren de base un sistema clásico por lo habituados que están a ese tipo de aprendizaje. Igualmente, el hecho de que el sistema de clase inversa haya sido implantado en esta asignatura recientemente puede hacer que los resultados en este grupo intermedio sean mejorables. 


\section{Estructura de la asignatura}

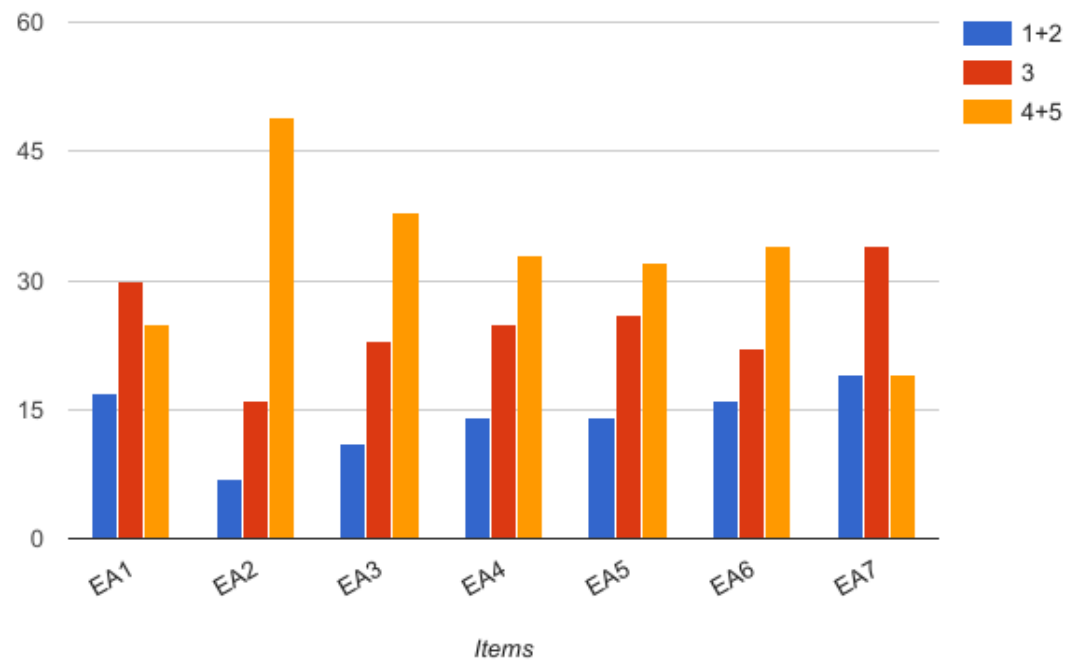

Fig. 1. Diagrama de barras que indica las puntuaciones de los items para la sección Estructura general de la asignatura. Se han sumado las puntuaciones 1 y 2 que muestran disconformidad (Muy en desacuerdo y En desacuerdo) así como las puntuaciones 4 y 5 que muestran conformidad (De acuerdo y Muy de acuerdo). Los items EA1 a EA7 están detallados en el apartado 3.1. En el eje de ordenadas se representa el número de alumnos que han respondido.

Finalmente, es de destacar que un $46 \%$ de los encuestados recomendaría este tipo de docencia frente a un $21 \%$ que no lo haría (EA6 Fig 1.). Igualmente, es interesante resaltar positivamente que nuestro sistema $\mathrm{NO}$ ha requerido más tiempo de dedicación a esta asignatura respecto a otras que comparte en el año docente (EA7 Fig 1.), indicando que este tipo de sistema es perfectamente integrable y realizable sin desequilibrar la carga docente y sin "monopolizar" a los alumnos para nuestra asignatura. Según nuestra encuesta, la relación profesor-alumno, curiosamente, no parece verse favorecida con este sistema (EA5 Fig 1.).

\subsection{La estructura en casos prácticos}

Los estudiantes fueron preguntados, de nuevo, si una estructura en casos prácticos ayuda a comprender y saber aplicar los contenidos, y los resultados son similares a los obtenido para las preguntas similares en el apartado anterior: como dato muy positivo, un $77 \%$ de los estudiantes piensa que este división las ayuda mucho a saber aplicar los contenidos (CP2 Fig. 2), ya que así pueden individualizar los conceptos y aplicarlos en casos tipo. Preguntados si con la lectura de artículos científicos (muchos casos plantean este tipo de ejercicios) habían conseguido perderle el miedo a lecturas de artículos, conseguimos nuestro objetivo con creces en un $40 \%$, mientras que un $36 \%$ todavía no han sido capaces de alcanzar este objetivo (CP3 Fig 2.). La lectura en un idioma extranjero (el $100 \%$ de los artículos propuestos están en inglés) puede ser una causa de este último porcentaje, lo que queda fuera de nuestros objetivos. 


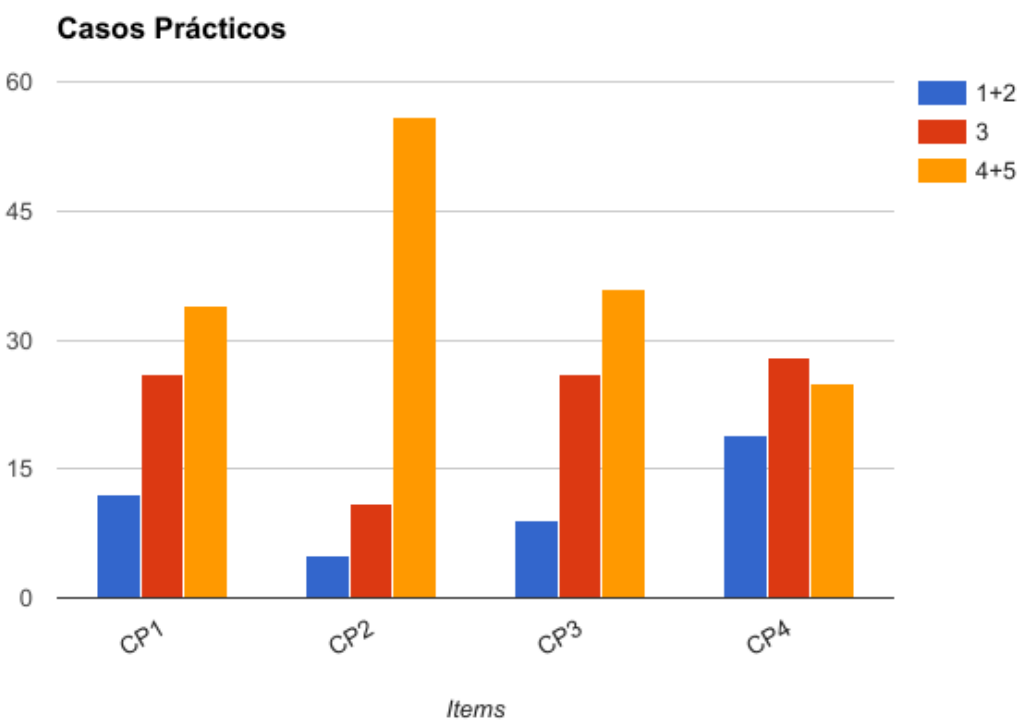

Fig. 2. Diagrama de barras que indica las puntuaciones de los items para la sección estructura general de la asignatura. Se han sumado las puntuaciones 1 y 2 que muestran disconformidad (Muy en desacuerdo y En desacuerdo) asi como las puntuaciones 4 y 5 que \%muestran conformidad (De acuerdo y Muy de acuerdo). Los items CP1 a CP4 están detallados en el apartado 3.1.En el eje de ordenadas se representa el número de alumnos que han respondido.

\subsection{Las tareas en casa (La clase inversa).}

El punto más conflictivo de la encuesta lo encontramos en la implantación del sistema de clase inversa. Preguntados si este tipo de clases con tareas en casa, anteriores o posteriores a los conceptos temáticos, que sirvan de base, o ayuden a consolidar los contenidos, había sido útil, las respuestas siguen una distribución normal centrada en el punto central (ni sí ni no), con un $30 \%$, un $27 \%$ en contra y el resto más indiferentes (T1 Fig 3.). Este es el primer año de la implantación en nuestra asignatura de la clase inversa, y quizá este resultado refleja las enormes posibilidades de mejora que tenemos todavía. Sin embargo, no perderemos de vista la posibilidad de que la clase inversa no sea considerada por los estudiantes como una mejor opción en lo que respecta a la realización de tareas en casa. Al mismo tiempo, un $48 \%$ de los estudiantes indican que la realización de estas tareas le ha quitado mucho tiempo de estudio (T2 Fig 3.), lo que quizá se refleja en la calidad de las respuestas, ya que hasta un $35 \%$ confiesa que no han respondido a las tareas como si fueran preguntas de examen, probablemente por la falta de tiempo en su realización (T3 Fig 3.). En años posteriores, consideramos que las tareas deberían ajustarse en tiempo y cantidad para evitar la saturación y conseguir una mayor implicación del alumno en las mismas. 


\section{Tareas}

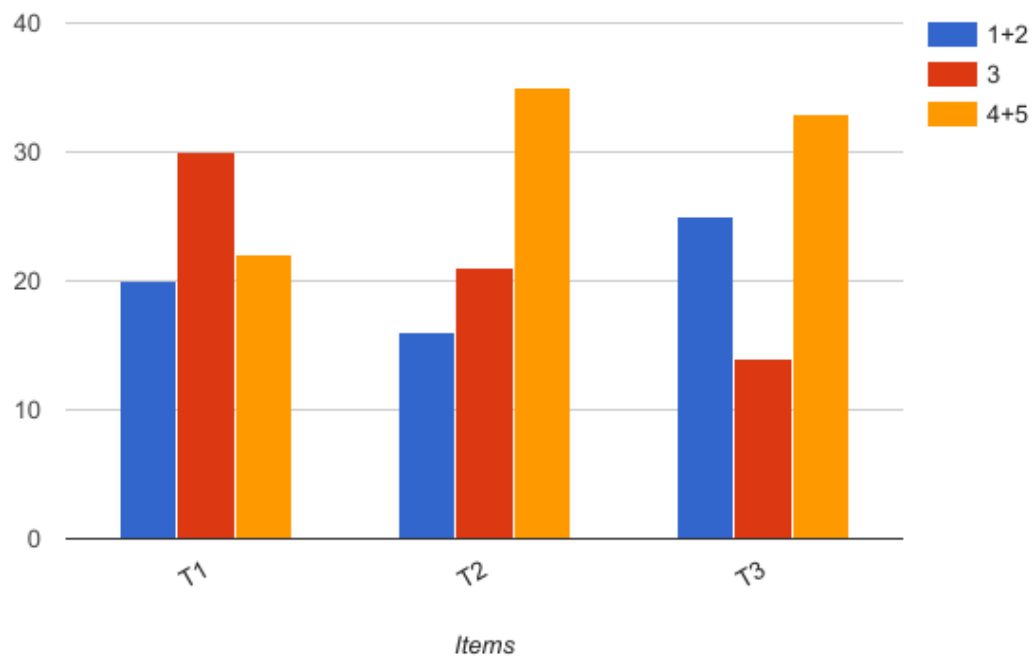

Fig. 3. Diagrama de barras que indica las puntuaciones de los items para la sección Tareas en casa. Se han sumado las puntuaciones 1 y 2 que muestran disconformidad (Muy en desacuerdo y En desacuerdo) así como las puntuaciones 4 y 5 que muestran conformidad (De acuerdo y Muy de acuerdo). Los items T1 a T3 están detallados en el apartado 3.1. En el eje de ordenadas se representa el número de alumnos que han respondido.

\subsection{Los trabajos/proyectos de investigación}

Este punto es con diferencia el aspecto más positivo de la asignatura. Implantados desde el año 2012-2013, estos trabajos han supuesto desde hace años un modo de involucrar a los estudiantes en proyectos casi reales, y el feedback recibido durante estos años ha sido muy positivo. Desgraciadamente, no tenemos encuestas de años anteriores, pero pretendemos enviar esta parte de la encuesta a los estudiantes de años anteriores para conocer la posible repercusión de haber realizado estos trabajos cuando los egresados ya están integrados en el mundo laboral.

Este tipo de trabajos serviría para evaluar la Competencia Transversal "Diseño de Proyectos". Los estudiantes fueron preguntados por aspectos como los siguientes: si les ha ayudado a discriminar información relevante e irrelevante para un determinado objetivo (P1 Fig 4.), si les ha ayudado a entender que pueden seguir adelante sin entender todo un proceso (P2 Fig 4.), si les ha ayudado a buscar e integrar diferentes fuentes para crear algo nuevo (P3 Fig 4.), si les ha ayudado a trabajar en grupo y a planificar su tiempo (P4,P5 y P6 Fig 4.), y si les ha motivado crear algo nuevo desde cero (P7 Fig 4.). Aunque los porcentajes individuales de cada pregunta pueden ser analizados con detalle, la tendencia general es muy positiva y nos ayuda a seguir con esta iniciativa original que, que sepamos, no realiza ninguna otra asignatura del actual plan de estudios. Un $60 \%$ reconoce que la realización de estos trabajos le ha motivado, frente a sólo un $16 \%$ que lo ve negativamente, y un $20 \%$ que está en un punto medio (P7 Fig 4.). Algunos porcentajes particularmente 
interesantes: más de un $60 \%$ considera que les ha ayudado a trabajar en grupo (P5 Fig 4.), un $72 \%$ indica que le ha ayudado a encontrar fuentes diversas y crear algo nuevo (P3 Fig 4.), o un $67 \%$ ha aprendido que no es necesario entenderlo todo al detalle para poder construir y entender un proyecto (P2 Fig 4.), importante para iniciales en la multidisciplinaridad de proyectos como los que se manejan en esta asignatura.

Finalmente, preguntados qué sentían mientras realizaban este proyecto, más de un $50 \%$ contestó esta opción: "Nunca he olvidado que era una obligación, pero encantado/a de que me obliguen a cosas asi!".

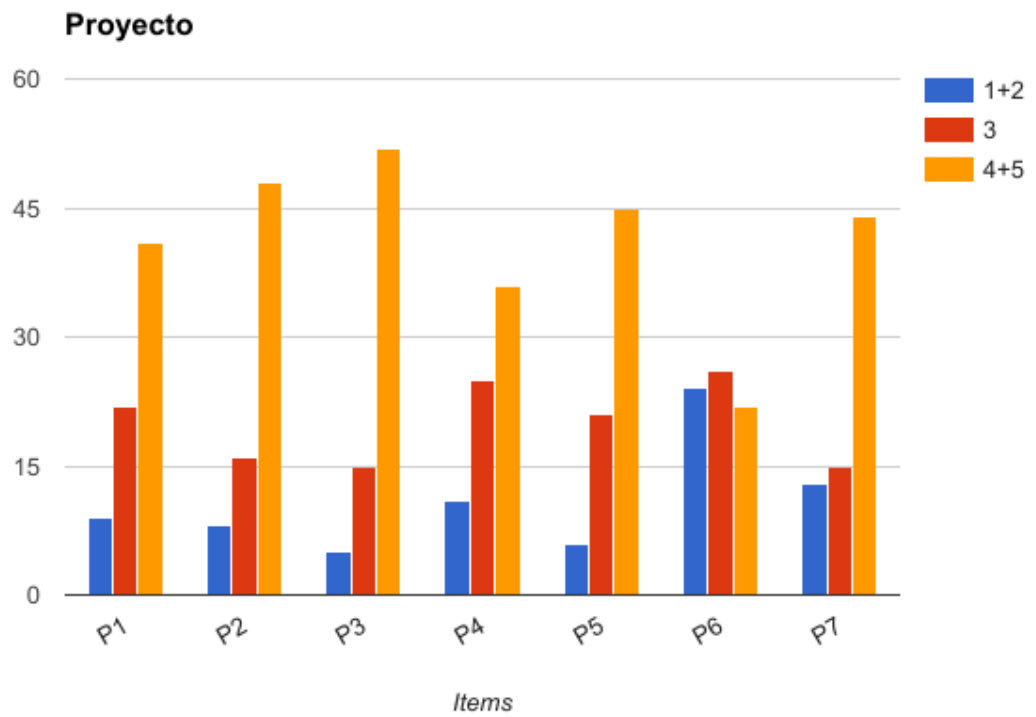

Fig. 4. Diagrama de barras que indica las puntuaciones de los items para la sección Proyecto de investigación. Se han sumado las puntuaciones 1 y 2 que muestran disconformidad (Muy en desacuerdo y En desacuerdo) así como las puntuaciones 4 y 5 que muestran conformidad (De acuerdo y Muy de acuerdo). Los items P1 a P7 están detallados en el apartado 3.1.En el eje de ordenadas se representa el número de alumnos que han respondido.

\subsection{Preferencias generales de los alumnos}

Analizando mediante un Heatmap los resultados en global se puede establecer que los alumnos caen en 3 categorías. Un pequeño grupo formado por 5 alumnos $(6,8 \%)$ que contestan Muy en desacuerdo y En desacuerdo a casi la totalidad de las preguntas (Cluster A Fig 5.). Un segundo grupo formado por 24 alumnos (32.9\%) cuyas respuestas en general fueron indiferentes (Cluster B Fig 5.). Por último, un grupo de 44 alumnos (60.3\%) cuyas respuestas en general apoyaron la metodología aplicada en clase (Cluster C Fig 5.). Esto indica que la mayoría de los alumnos ve aspectos positivos en esta manera de impartir las clases. 


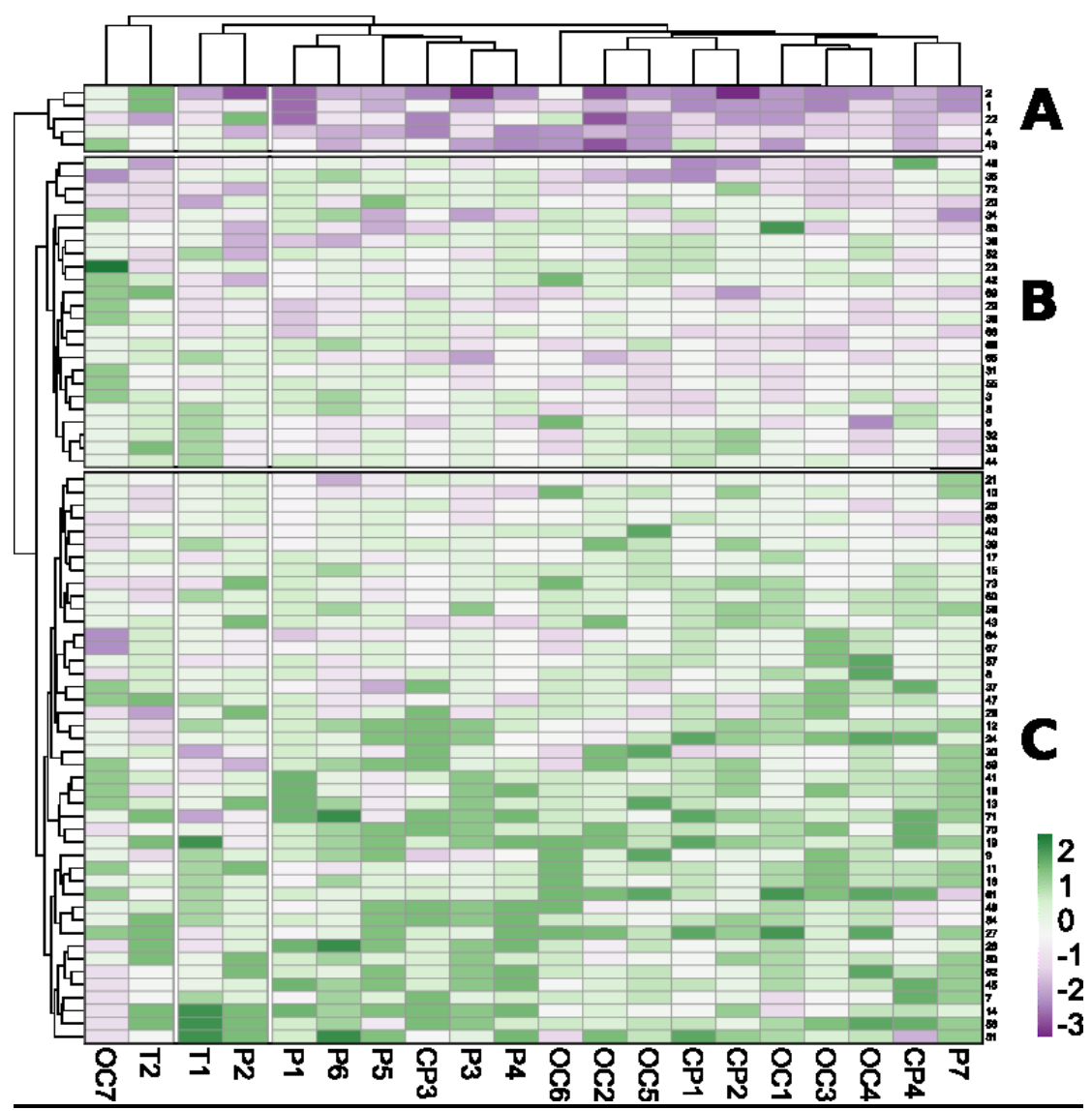

Fig. 5. Heatmap para los items evaluados (columnas) y todos los alumnos (filas). El programa indica la existencia de tres clusters de alumnos $(A, B y C)$.

\section{Conclusiones}

De modo general, estos resultados nos animan a continuar con el sistema docente que hemos implantado en esta asignatura del grado de Biotecnología, y que alterna clases magistrales, trabajos de investigación y clase inversa. Con seguridad, muchos aspectos de la misma, a raíz de los resultados obtenidos, son susceptibles de mejora. Los más mejorables apuntan sin duda a la implantación y ejecución de la clase inversa, un proyecto piloto que hemos iniciado este año y que lógicamente requiere un período de puesta a punto para que encaje con nuestros objetivos y con el tipo de asignatura, y que al mismo tiempo sea atractivo para el alumno. Los aspectos que ya parecen más enraizados y que según la encuesta parece ser una excelente iniciativa son los proyectos de investigación aplicada, implantados ya desde hace unos años pero sólo éste hemos elaborado encuestas que evalúen de modo estadístico la opinión de los alumnos.

En resumen, la implantación de estrategias de aprendizaje autónomo que ponga énfasis en un aprendizaje más aplicado, que aumente la responsabilidad por parte del estudiante e incremente su sentido de autonomía, es visto con buenos ojos por una alta proporción del 
alumnado y podría ser implantado de un modo organizado en los planes docentes, sustituyendo o alternando con estrategias docentes más tradicionales.

\section{Referencias}

BRIONES, G. (1995). Métodos y Técnicas de Investigación para las Ciencias Sociales,2a . reimp., Ed. Trillas, México.

CRISPIN BERNARDO, MARÍA LUISA, AND MA. DEL CARMEN DORIA SERRANO (2011). Aprendizaje autónomo orientaciones para la docencia. México: Universidad Iberoamericana. http://www.uia.mx/web/files/publicaciones/aprendizaje-autonomo.pdf.

METSALU, T., VILO, J. (2015). ClustVis: a web tool for visualizing clustering of multivariate data using Principal Component Analysis and heatmap. Nucleic Acids Research, 43, W566-W570. http://doi.org/10.1093/nar/gkv468

NORTHWEST REGIONAL EDUCATIONAL LABORATORY (2006). Aprendizaje por proyectos. http://www.eduteka.org/AprendizajePorProyectos.php; ABP en Eduteka. [Consultado el 25 de Marzo de 2017]. 